\title{
Average Bit Error Rate at Signal Transmission with OOK Modulation Scheme in Different FSO Channels
}

\author{
Jelena TODOROVIĆ*, Branimir JAKŠIĆ, Petar SPALEVIĆ, Djoko BANDJUR, Stefan PANIĆ
}

\begin{abstract}
In this paper, the Average Bit Error Rate of the signal in the Free Space Optical system modulated with On-Off keying scheme is calculated and analysed. The Average Bit Error Rate is determined in the case of an atmospheric channel modelled with Gamma-Gamma distribution, Log-Normal distribution, $K$ distribution and $I-K$ distribution. The results are presented both analytically and graphically for different lengths of the Free Space Optical link and the strength of the atmospheric turbulence. The quality of the received signal based on the Average Bit Error Rate for weak, moderate and strong atmospheric turbulences, different lengths of the transmission section and different Signal-to-Noise Ratio values was analysed. The operation of the Free Space Optical system in the observed environment was simulated and the transmission quality was analysed based on Bit Error Rate and $Q$ factor.
\end{abstract}

Keywords: ABER - Average Bit Error Rate; Gamma-Gamma distribution; I-K distribution; K distribution; Log-Normal distribution; OOK - On-Off keying

\section{INTRODUCTION}

Continuous development of various wireless telecommunication system services leads to the need to study and improve their performance. The basic requirements that are present in the process of improving the performance of wireless telecommunication systems are: providing high transmission speeds, large channel capacity and as greater connection range as possible with the lowest possible bit error rate.

Free Space Optical (FSO) is a communication technology that provides full-duplex wireless gigabit data transmission $[1,2]$. FSO systems allow digital signal transmission with a bandwidth of several $\mathrm{Gb} / \mathrm{s}$, while microwave connections allow bandwidth of several $\mathrm{Mb} / \mathrm{s}$. FSO system uses a wide frequency spectrum. It is resistant to electromagnetic perturbation and interference with adjacent channels, due to a well defined narrow signal beam without power attenuation. Moreover, it provides a high level of safety $[1,3]$. FSO links are short and range up to $3.5 \mathrm{~km}$. The greatest impact on the quality of a transmitted signal comes from the atmospheric conditions, though with some variations. Thus, snow and rain have a considerably lower impact on the transmission signal quality than the atmospheric turbulences and fog. In general, the atmospheric conditions impact on FSO systems is noticeable through phenomena such as: the beam spread, intensity fluctuation and signal phase, scintillation [4].

The signal propagation in a FSO system, in the presence of atmospheric turbulence, can be characterized by a number of statistical models. Some of them are: Gamma-Gamma, Log-Normal, Negative Exponential, $K$ distribution, $I-K$ distribution, IG distribution, DoubleWeibull, Exponentiated Weibull, and Double Generalized distribution [5].

A FSO system performances are defined by a number of parameters including: Signal to Noise Ratio (SNR), Outage Probability (OP), Channel Capacity (CC) and Average Bit Error Rate (ABER) [6]. To determine the ABER, it is necessary to know the Probability Density Function (PDF) of the received signal, which is nonstationary by nature and depends on the parameters related to atmospheric turbulence. Thus, the ABER is a good indicator of the received signal quality in FSO systems [7].
Several modulation formats are used for transmitting signals in FSO systems, including the most popular On-Off Keying (OOK), Binary Phase Shift Keying (BPSK) and Differential Phase Shift Keying (DPSK). The BPSK, requires a more complex implementation and shows much better performances compared to DPSK. On the other hand, DPSK shows better performance compared to OOK [8].

In its simplest form, FSO systems use the OOK modulation scheme, where binary " 1 " and binary " 0 " are represented by the presence and absence of the optical impulse, respectively. The simplicity of the OOK modulation scheme is reflected in the fact that the optical impulse representing the binary symbol "1" takes the entire signaling interval. Intensity Modulation/Direct Detection (IM/DD) using OOK is the simplest scheme and is widely applied in the commercially available FSO systems. OOK is very sensitive to channel turbulence. The threshold level in a standard OOK-FSO system is usually fixed at the half of the range between the expected levels of the binary symbols $[9,10]$.

Non-Return-to-Zero (NRZ) and Return-to-Zero (RZ) OOK models are often used within the OOK modulation scheme due to their easy implementation and cost effectiveness. The RZ OOK modulation performs better than the NRZ OOK because it generally has a lower BER, a higher SNR, a better nonlinear tolerance and power efficiency and it offers a longer transmission distance compared to NRZ. NRZ OOK provides high bandwidth efficiency $[11,12]$.

Statistical characterization of a FSO system modelled with the Gamma-Gamma and Negative Exponential distributions are presented in [7] and [13], respectively. The ABER of a FSO channel modelled with GammaGamma distribution is shown in [14]. Some of the OOK modulation scheme characteristics in a FSO system are analyzed in [15]. The ABER for other modulation formats is considered in [16-18]. Comparison of performance analysis of FSO and Single Mode Fiber based on the $Q$ Factor and BER done by OptiSystem simulations is presented in [19], while in [20] and [21] is given performance analysis of fiber optical network based on WDM technology.

In this paper, the ABER expressions for GammaGamma, Log-Normal, $K$, and $I-K$ distributions are derived 
for FSO system using the OOK modulation scheme. In Section 2 is given the analyzed system model, and the initial expressions for the ABER calculation. In Section 3, analytical expressions for the ABER for all four above mentioned distributions are derived and presented. The obtained numerical results are presented and discussed in Section 4, for different levels of atmospheric turbulence and length of the FSO link. In Section 5 is shown a simulation of the FSO system operation with RZ OOK and NRZ OOK modulation scheme in the OptiSystem environment, which was analyzed for different lengths of the FSO link, as well as for attenuations caused by the impact of various atmospheric phenomena. Section 6, summarizes the conclusions.

\section{SYSTEM MODEL}

A typical FSO system is considered in this paper. As shown in Fig. 1, it consists of the following components: modulator, transmitter, atmospheric channel, receiver, and demodulator.

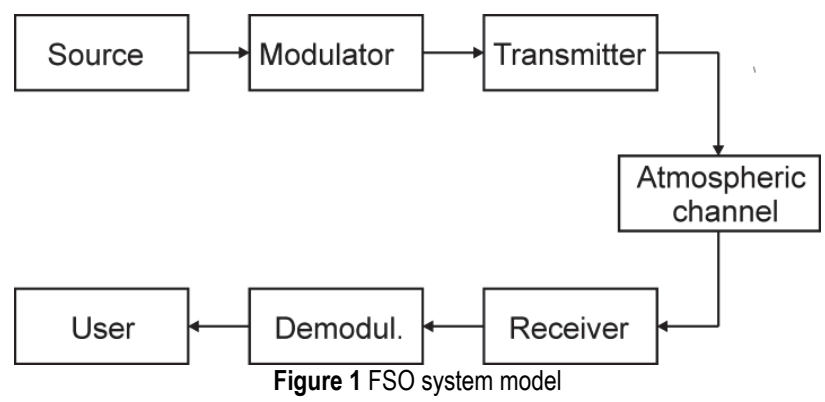

The digital input signal, generated by the source, is modulated in the modulator by using the OOK modulation scheme. The modulated signal is then transmitted through an atmospheric channel modeled with Gamma-Gamma, Log-Normal, $K$ and $I-K$ distributions. For all four atmospheric channel models, the ABER is determined at the receiving side of the system.

The PDF for the Gamma-Gamma model is given by the expression [14]:

$$
f_{I}(I)=\frac{2(\alpha \beta)^{\frac{\alpha+\beta}{2}}}{\Gamma(\alpha) \Gamma(\beta)} I^{\frac{\alpha+\beta}{2}-1} K_{\alpha-\beta}(2 \sqrt{\alpha \beta I})
$$

where $I$ is irradiance at the receiver, $\Gamma(\cdot)$ represents the Gamma function [22, Eq. 8.310], and $K_{v}(\cdot)$ th-order modified Bessel function of the second kind [22, Eq. 8.432]. The parameters $\alpha$ and $\beta$ are the effective numbers of small-scale and large-scale eddies of the scattering environment, respectively. These are parameters of the atmospheric turbulence that for propagation of plane waves and zero inner scale can be expressed as [14]:

$\alpha=\left[\mathrm{e}^{\frac{0.49 \sigma_{\mathrm{R}}^{2}}{\left(1+1.11 \sigma_{\mathrm{R}}^{12 / 5}\right)^{7 / 6}}}-1\right]^{-1}$ $\beta=\left[\mathrm{e}^{\frac{0.51 \sigma_{\mathrm{R}}^{2}}{\left(1+0.69 \sigma_{\mathrm{R}}^{12 / 5}\right)^{5 / 6}}}-1\right]^{-1}$

where $\sigma_{\mathrm{R}}^{2}$ represents the Rytov variance used to determine the intensity of the optical signal due to atmospheric turbulence, and is defined as:

$\sigma_{\mathrm{R}}^{2}=1.23 C_{n}^{2} k^{7 / 6} L^{11 / 6}$

The parameter $C_{n}^{2}$ denotes the index of refraction used as a measure of the turbulence strength. For the horizontal propagation path, the parameter $C_{n}^{2}$ is considered constant with mean values from $10^{-17} \mathrm{~m}^{-2 / 3}$ to $10^{-13} \mathrm{~m}^{-2 / 3}$ for channels from weak to strong turbulence, respectively. The parameter $k$ is an optical wave number, defined as $k=2 \pi / \lambda$ with wavelength $\lambda$, while $L$ is distance between the transmitter and the receiver, i.e. the length of the optical signal propagation. The Gamma-Gamma distribution is used for modeling the atmospheric channel in a weak, moderate and strong atmospheric turbulence [5].

The Log-Normal distribution of the irradiance at the receiver is given by [23]:

$$
f_{I}(I)=\frac{1}{\sqrt{2 \pi \sigma^{2}}} \frac{1}{I} \exp \left(-\frac{\left(\ln (I)+\frac{\sigma^{2}}{2}\right)^{2}}{2 \sigma^{2}}\right)
$$

The parameter $\sigma$ is the scintillation index defined by Eq. (4) [24]. The Log-Normal distribution is used for modeling the atmospheric channel in a weak and moderate atmospheric turbulence [5].

The $K$ distribution represents a product of the exponential and Gamma distributions [5]. The $K$ distribution of the irradiance at the receiver is given by [25]:

$f_{I}(I)=\frac{2 \alpha^{\frac{\alpha+1}{2}}}{\Gamma(\alpha)} I^{\frac{\alpha-1}{2}} K_{\alpha-1}(2 \sqrt{\alpha I})$

where the parameter $\alpha$ is defined by Eq. (2). The $K$ distribution is used for modeling the atmospheric channel in a strong atmospheric turbulence [5].

A generalized form of the $K$ distribution applicable to all atmospheric turbulence conditions, including weak turbulence for which the $K$ distribution is not suitable, is the $I-K$ distribution. For the $I-K$ distribution, the field of the optical wave is modeled as the sum of a coherent (deterministic) component and a random component [5].

The $I-K$ distributed of the irradiance at the receiver is given by [25]: 


$$
f_{I}(I)=\left\{\begin{array}{lc}
2 \alpha(1+\rho)\left(1+\frac{1}{\rho}\right)^{\frac{\alpha-1}{2}} I^{\frac{\alpha-1}{2}} K_{\alpha-1}(2 \sqrt{\alpha \rho}) \times \\
\times I_{\alpha-1}(2 \sqrt{\alpha(1+\rho) I}), & 0<I<\frac{\rho}{1+\rho} \\
2 \alpha(1+\rho)\left(1+\frac{1}{\rho}\right)^{\frac{\alpha-1}{2}} & I^{\frac{\alpha-1}{2}} I_{\alpha-1}(2 \sqrt{\alpha \rho}) \times \\
\times K_{\alpha-1}(2 \sqrt{\alpha(1+\rho) I}), & I>\frac{\rho}{1+\rho}
\end{array}\right.
$$

where $\rho$ is the coherence parameter, and $I_{v}(\cdot) v$ th-order modified Bessel function of the first kind [22, Eq. 8.431]. The parameter $\alpha$ is defined by Eq. (2). The $I-K$ distribution is used for modeling the atmospheric channel in a weak and strong atmospheric turbulence [5].

The Average Bit Error Rate which depends on the irradiance fluctuation at the receiver for an optical signal transmitted by FSO system with the OOK modulation scheme can be expressed as [9]:

$$
P_{e}=\frac{1}{2} \int_{0}^{\infty} \operatorname{erfc}\left(\frac{\eta I}{2 \sqrt{N_{0}}}\right) f_{I}(I) \mathrm{d} I
$$

where $\eta$ represents the optical to-electrical conversion coefficient, and $N_{0}$ the mean noise optical power. The function $f_{I}(I)$ represents PDF of the received signal, i.e. the received irradiance fluctuation, and is used for atmospheric turbulence channel modeling.

\section{ANALYTICAL RESULTS \\ 3.1 Gamma-Gamma Distribution}

In order to derive a closed-form expression for the ABER, the modified Bessel function of the second kind $K_{v}(\cdot)$ in Eq. (1) is represented by the Meijer $G$ function as [22, Eq. 9.34.3]:

$K_{v}(x)=\frac{1}{2} G_{0,2}^{2,0}\left[\left.\frac{x^{2}}{4}\right|_{(v-2),-(v-2)} ^{-}\right]$

and the complementary error function $\operatorname{erfc}(\cdot)$ in Eq. (8) is represented by the Meijer $G$ function as [26, Eq. 06.27.26.0003.01]:

$$
\operatorname{erfc}(x)=1-\frac{x}{\sqrt{\pi}} G_{1,2}^{1,1}\left[x^{2} \mid \begin{array}{c}
1 / 2 \\
0,
\end{array}\right]
$$

By substituting Eq. (1) into Eq. (8), and making use of the relationships given in Eq. (9) and Eq. (10), as well as by using the solutions of the resulting integral given by [22, Eq. 7.811.4] and [27, Eq. 07.34.21.0012.01], we derive the ABER closed form expression as follows:

$$
\begin{aligned}
& P_{e}=\frac{1}{2}-\frac{1}{4 \sqrt{\pi} \alpha \beta \Gamma(\alpha) \Gamma(\beta)} \frac{\eta}{\sqrt{N_{0}}} \times \\
& \times H_{3,2}^{1,3}\left[\left(\frac{\eta}{2 \alpha \beta \sqrt{N_{0}}}\right)^{2}\left(\begin{array}{c}
\left(\frac{1}{2}, 1\right),(-\alpha, 2),(-\beta, 2) \\
(0,1),\left(-\frac{1}{2}, 1\right)
\end{array}\right]\right.
\end{aligned}
$$

\subsection{Log-Normal Distribution}

By substituting Eq. (5) into Eq. (8), by making use of the variable change $t=\left(\ln (I)+\sigma^{2} / 2\right) / \sqrt{2} \sigma$ and identity [26, Eq. 06.27.02.0001.01]:

$\operatorname{erfc}(x)=1-\frac{2}{\sqrt{\pi}} \sum_{n=0}^{\infty} \frac{(-1)^{n} \cdot x^{2 n+1}}{n ! \cdot(2 n+1)}$

as well as by using the solutions of the resulting integral given by [28, Eq. 01.03.21.0029.01] and [22, Eq. 3.322.2], we derive the ABER expression as follows:

$$
\begin{aligned}
& P_{e}=\frac{1}{4}-\frac{1}{2 \sqrt{\pi}} \sum_{n=0}^{\infty} \frac{(-1)^{n}}{n !(2 n+1)}\left(\frac{\eta}{2 \sqrt{N_{0}}}\right)^{2 n+1} \times \\
& \times \exp \left(\sigma_{i}^{2} n(2 n+1)\right)\left[1-\operatorname{erf}\left(-\frac{\sqrt{2}}{2} \sigma_{i}(2 n+1)\right)\right]
\end{aligned}
$$

\subsection{K Distribution}

By substituting Eq. (6) into Eq. (8), by making use of the relationships given in Eq. (9) and Eq. (10), as well as by using the solutions of the resulting integral given by [22, Eq. 7.811.4] and [27, Eq. 07.34.21.0012.01], we derive the ABER expression as follows:

$$
\begin{aligned}
& P_{e}=\frac{1}{2}-\frac{1}{4 \sqrt{\pi} \alpha \Gamma(\alpha)} \frac{\eta}{\sqrt{N_{0}}} \times \\
& \times H_{3,2}^{1,3}\left[\left(\frac{\eta}{2 \alpha \sqrt{N_{0}}}\right)^{2}\left[\begin{array}{c}
\left(\frac{1}{2}, 1\right),(-\alpha, 2),(-1,2) \\
(0,1),\left(-\frac{1}{2}, 1\right)
\end{array}\right]\right.
\end{aligned}
$$

\subsection{I-K Distribution}

By substituting Eq. (7) for $0<I<\rho /(1+\rho)$ into Eq. (8) and expressing the modified Bessel function of the first kind $I_{v}(\cdot)$ in Eq. (7) in the form of an infinite series [22, Eq. 8.445]:

$I_{v}(x)=\sum_{n=0}^{\infty} \frac{1}{n ! \cdot \Gamma(v+n+1)}\left(\frac{x}{2}\right)^{v+2 n}$

as well as by using the solution of the resulting integral given by [22, Eq. 7.811.4], we derive the ABER expression as follows: 
$P_{e}=\sum_{n=0}^{\infty} \frac{1}{2 \sqrt{\pi} n !} \frac{\Gamma\left(\frac{\alpha+n}{2}\right) \Gamma\left(\frac{\alpha+n+1}{2}\right)}{\Gamma(\alpha+n) \Gamma\left(\frac{\alpha+n+2}{2}\right)}\left(1+\frac{1}{\rho}\right)^{\frac{\alpha-1}{2}} \times$

$\times(\alpha(1+\rho))^{\frac{\alpha+1}{2}+n}\left(\frac{2 \sqrt{N_{0}}}{\eta}\right)^{\alpha+n} K_{\alpha-1}(2 \sqrt{\alpha \rho})$

By substituting Eq. (7) for $I>\rho /(1+\rho)$ into Eq. (8) by using Eq. (9) and Eq. (10), and solution of the resulting integral given by [27, Eq. 07.34.21.0012.01], we derive the ABER expression as follows:

$$
\begin{aligned}
& P_{e}=\frac{1}{2} \Gamma(\alpha)(\alpha(1+\rho))^{-\frac{\alpha-1}{2}}\left(1+\frac{1}{\rho}\right)^{\frac{\alpha-1}{2}} I_{\alpha-1}(2 \sqrt{\alpha \rho})- \\
& -\frac{\eta}{4 \sqrt{\pi} \sqrt{N_{0}}}(\alpha(1+\rho))^{-\frac{\alpha+1}{2}}\left(1+\frac{1}{\rho}\right)^{\frac{\alpha-1}{2}} I_{\alpha-1}(2 \sqrt{\alpha \rho}) \times \\
& \times H_{3,2}^{1,3}\left[\left(\frac{\eta}{2(\alpha(1+\rho)) \sqrt{N_{0}}}\right)^{2}\left(\begin{array}{c}
\left(\frac{1}{2}, 1\right),(-\alpha, 2),(-1,2) \\
(0,1),\left(-\frac{1}{2}, 1\right)
\end{array}\right]\right.
\end{aligned}
$$

\section{NUMERICAL RESULTS}

For the numerical calculation, the FSO system was observed at a wavelength $\lambda=1550 \mathrm{~nm}$. Three types of atmospheric turbulence were considered: weak, moderate and strong, with indexes of reflection $C_{n}^{2}=6 \times 10^{-15} \mathrm{~m}^{-2 / 3}$, $C_{n}^{2}=2 \times 10^{-14} \mathrm{~m}^{-2 / 3}$ and $C_{n}^{2}=1.2 \times 10^{-13} \mathrm{~m}^{-2 / 3}$, respectively. In terms of the distance between transmitter and receiver, two cases were observed: $L=1 \mathrm{~km}$ and $L=3 \mathrm{~km}$. Values of the parameters $\sigma_{R}^{2}, \alpha$ and $\beta$ obtained for the considered cases are given in Tab. 1 .

Table 1 Values of atmospheric turbulence parameters
\begin{tabular}{|c|c|c|c|c|}
\hline \multicolumn{2}{|c|}{$C_{n}{ }^{2}$} & $6 \times 10^{-15}$ & $2 \times 10^{-14}$ & $1.2 \times 10^{-13}$ \\
\hline \multirow{3}{*}{$L=1 \mathrm{~km}$} & $\sigma_{R}{ }^{2}$ & 0,12 & 0,4 & 2,39 \\
\cline { 2 - 5 } & $\alpha$ & 18,33 & 6,9 & 4,02 \\
\cline { 2 - 5 } & $\beta$ & 16,65 & 5,36 & 1,57 \\
\hline \multirow{3}{*}{$L=3 \mathrm{~km}$} & $\sigma_{R}{ }^{2}$ & 0,89 & 2,98 & 17,9 \\
\cline { 2 - 5 } & $\alpha$ & 4,55 & 4,12 & 7,06 \\
\cline { 2 - 5 } & $\beta$ & 2,77 & 1,44 & 1,05 \\
\hline
\end{tabular}

The ABER as a function of the electrical SNR (Signal to Noise Ratio), for various atmospheric channel models and OOK modulation scheme expressed by Eq. (11), Eq. (13), Eq. (14), and Eq. (17) is graphically presented in Figs. 2, 3, 4 and 5. Electrical SNR is defined as $\mu=\eta^{2} E[I] / N_{0}$

[9]. Without loss of generality, it is assumed that $E[I]=1$ [9]. It can be seen from the given figures that the ABER decreases as the SNR increases. Also, as expected, for longer link distances and stronger turbulence, the higher ABER values are obtained.

Fig. 2 indicates that in the case of Gamma-Gamma channel model, with applied OOK modulation scheme, the ABER decreases significantly faster for shorter distances than for those longer ones. A similar conclusion can be obtained if the turbulence strength is observed. Namely, the ABER decreases significantly faster for lower than for higher turbulence levels.

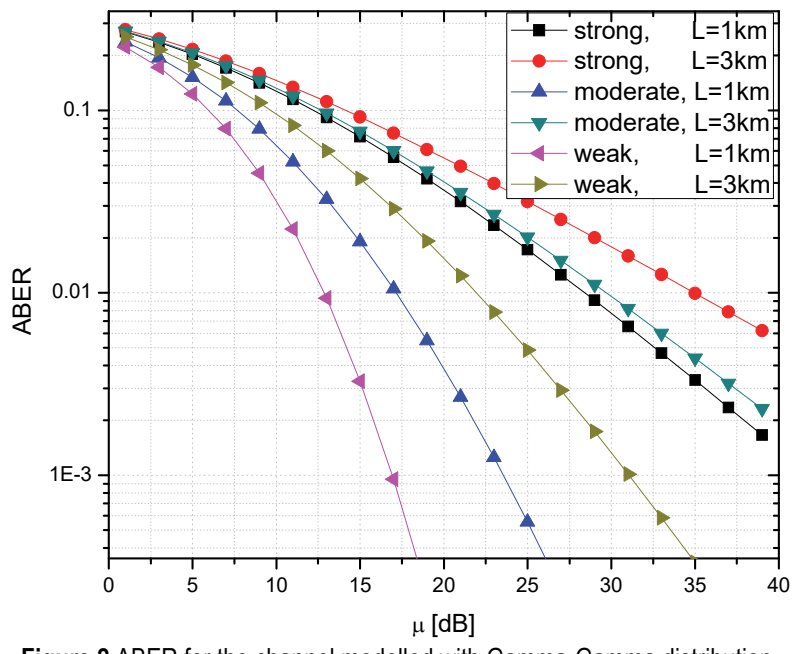

Figure 2 ABER for the channel modelled with Gamma-Gamma distribution

Fig. 3 indicates that the ABER behavior for the atmospheric channel modelled with the Log-Normal distribution is almost identical to the one with the GammaGamma distribution. The only deviation can be seen in the case of strong atmospheric turbulence, where the values of the ABER are extremely high. For this reason, the LogNormal distribution is not used for modelling the FSO channel in strong turbulence.

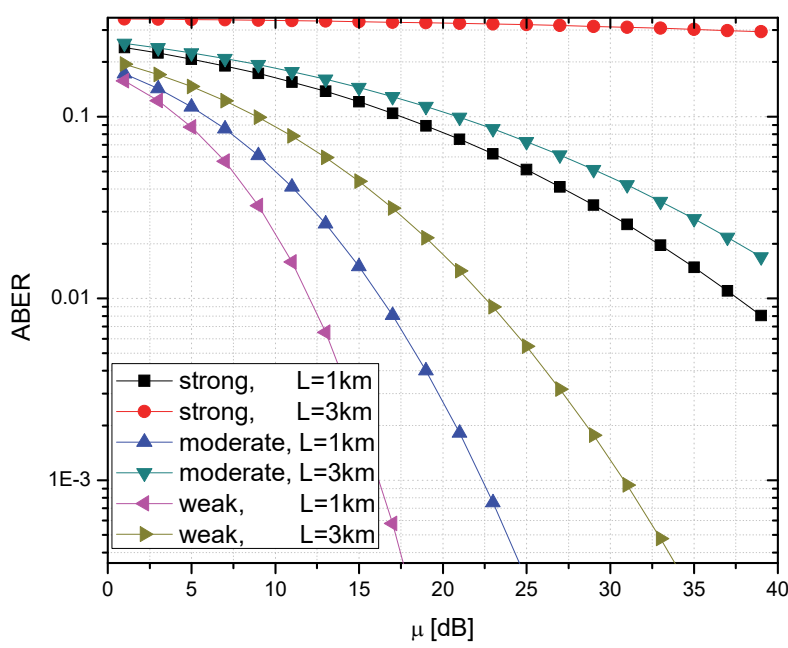

Figure 3 ABER for the channel modelled with Log-Normal distribution

From Fig. 4, it can be seen that the atmospheric channel modeled with the $K$ distribution gives approximately the same values for all levels of atmospheric turbulence and the length of the FSO link. Also, the ABER change is approximately linear with respect to the other distributions considered in this paper.

A very important fact that can be observed with regard to $K$ distribution is that in the case of lower turbulence levels a slightly worse results are obtained compared to strong turbulence. Thus, this distribution is used exclusively for modeling channels in strong turbulence. 


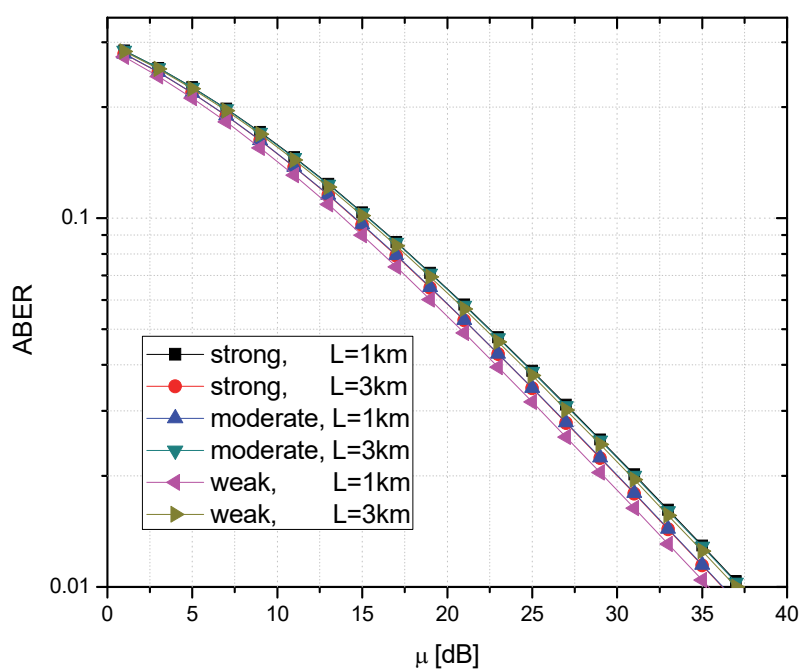

Figure 4 ABER for the channel modelled with $\mathrm{K}$ distribution

From Fig. 5, it can be seen that in the case of atmospheric channel modelled with $I-K$ distribution, the differences between ABER at different levels of turbulence are significantly less than in atmospheric channels modelled with Gamma-Gamma and Log-Normal distributions. Generally, up to $\mu=10 \mathrm{~dB}$, the obtained numerical ABER values are very similar. The worst results are obtained in the case of moderate atmospheric turbulence channel. Thus, the $I-K$ distribution model should be exclusively used for modelling the FSO channel in weak and strong atmospheric turbulences.

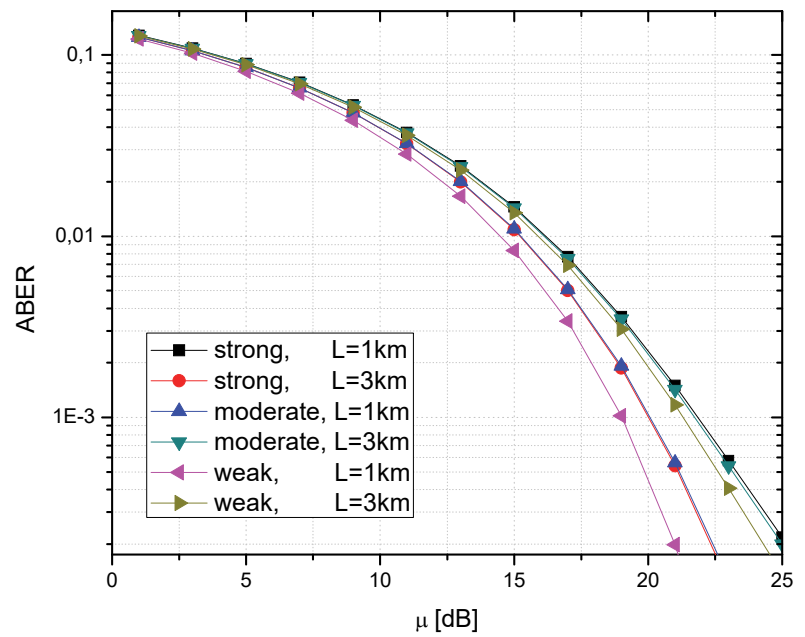

Figure 5 ABER for the channel modelled with $I-K$ distribution

In Fig. 6, Fig. 7 and Fig. 8, are given comparisons of the ABER for different distributions in the cases of strong, moderate, and weak turbulence, respectively. From the given figures, it can be seen that an increase in the length of the FSO link leads to an increase in the ABER, with an exception that this increase has different effects in different distribution models.

From Fig. 6, it can be seen that in case of strong atmospheric turbulence, the best results are achieved by the Gamma-Gamma distribution, for a shorter length of the FSO link, up to $2 \mathrm{~km}$. The $K$ and $I-K$ distributions give the approximately constant ABER values over the entire length of the FSO link. Thus, for longer sections it is more adequate to model atmospheric channels with the $K$ and $I$ $K$ distributions.

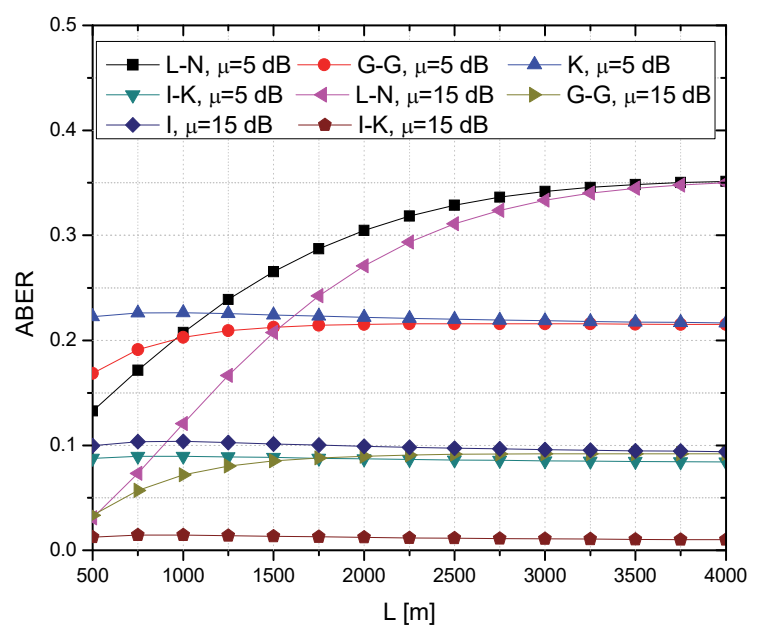

Figure 6 ABER for different distributions in the case of strong turbulences

From Fig. 7, it can be seen that in moderate atmospheric turbulence, the best results are given by the Log-Normal and Gamma-Gamma distributions, while the $K$ distribution gives the worst results.

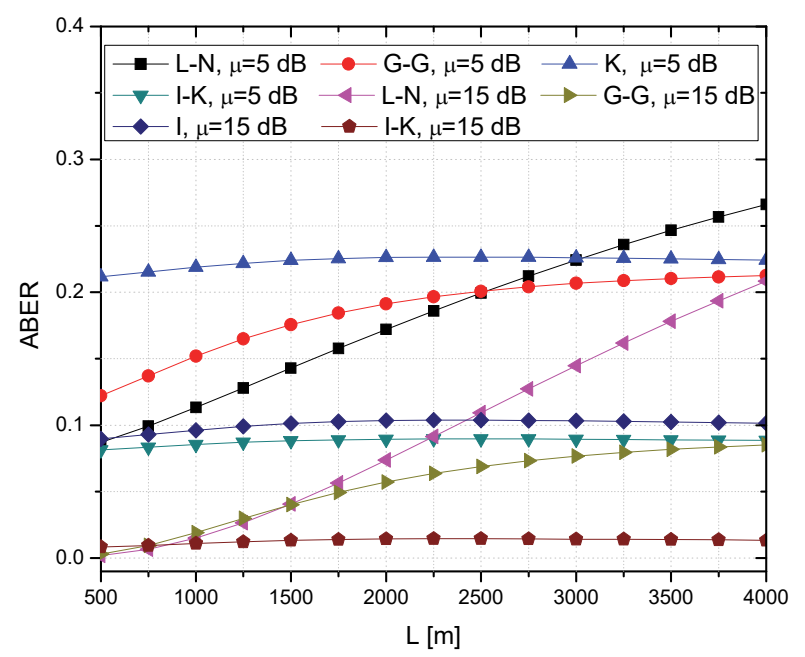

Figure 7 ABER for different distributions in the case of moderate turbulences

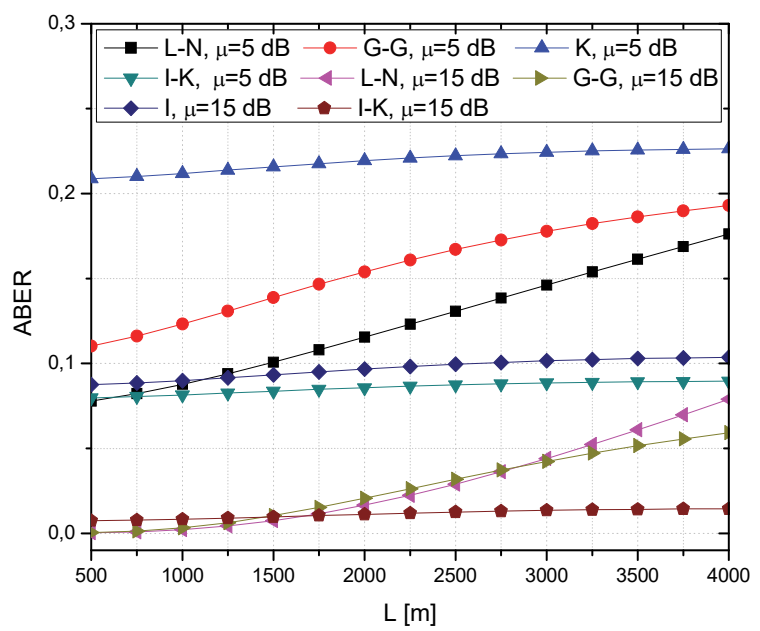

Figure 8 ABER for different distributions in the case of weak turbulences

From Fig. 8, it can be concluded that in the case of weak atmospheric turbulence, as well as in the case of 
moderate, the worst results are given by the $K$ distribution. From that reason, it is not suitable for modeling the FSO channel in weak atmospheric turbulence. The satisfying results are achived by the $I-K$, Log-Normal and GammaGamma distributions. However, an increase of the ABER due to increased length of the FSO link is much more pronounced in the case of Log-Normal and GammaGamma distributions than in the case of $I-K$ distribution.

\section{SIMULATION RESULTS}

The simulation of the FSO system operation shown in Fig. 1 was performed using the OptiSystem 7.4 software package [29]. The simulation system consists of a PseudoRandom Bit Sequence and a Pulse Generator (RZ and NRZ) which are fed together to a Mach-Zehnder Modulator. The modulated signal is sent to the FSO channel where a certain level of attenuation due to atmospheric impacts is introduced. On the receiving side there is an Optical Receiver with Cutoff Frequency = $0.75^{*}$ BitRate $\mathrm{Hz}$ and a BER Analyzer for reading the measured parameters. The system was simulated at a wavelength of $1550 \mathrm{~nm}$ and a transmitter power of $10 \mathrm{~mW}$.

The FSO system was analysed on link distance of 1000 $\mathrm{m}, 1500 \mathrm{~m}, 2000 \mathrm{~m}, 2500 \mathrm{~m}$ and $3000 \mathrm{~m}$, as well as for attenuations caused by the impact of various atmospheric phenomena: Clear, Light haze, Heavy haze, Light rain, Medium rain and Heavy rain, whose attenuations are of $0.233 \mathrm{~dB} / \mathrm{km}, 0.55 \mathrm{~dB} / \mathrm{km}, 2.37 \mathrm{~dB} / \mathrm{km}, 6.27 \mathrm{~dB} / \mathrm{km}, 9.64$ $\mathrm{dB} / \mathrm{km}$ and $19.28 \mathrm{~dB} / \mathrm{km}$, respectively [30]. Attenuations caused by the atmospheric phenomenon Clear and Light haze can be classified in the category of Weak turbulence, Heave haze and Light rain in the category of Moderate turbulence, Medium rain and Heavy rain in the category of Strong turbulence. Two cases of OOK modulation scheme were considered - RZ and NRZ.

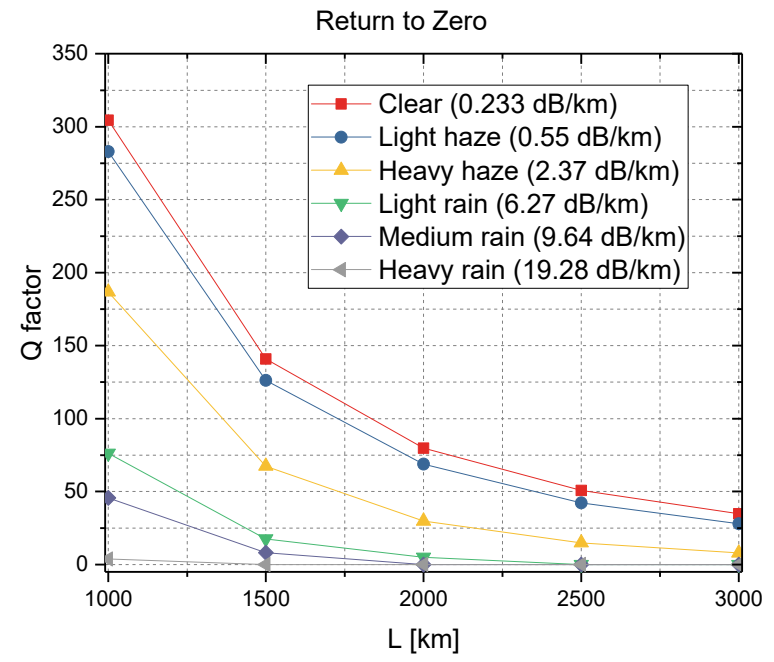

Figure $9 \mathrm{Q}$ factor for $\mathrm{RZ}$ OOK modulation

Fig. 9 and Fig. 10 show the change of $Q$ factor versus the length of the link distance and for attenuations caused by various atmospheric phenomena, for RZ OOK and NRZ OOK modulation scheme, respectively. BER eye diagrams for link distance $L=2000 \mathrm{~m}$, RZ OOK and NRZ OOK modulation scheme and attenuation caused by Clear,
Heavy haze and Medium rain are given in Fig. 11, Fig. 12 and Fig. 13, respectively.

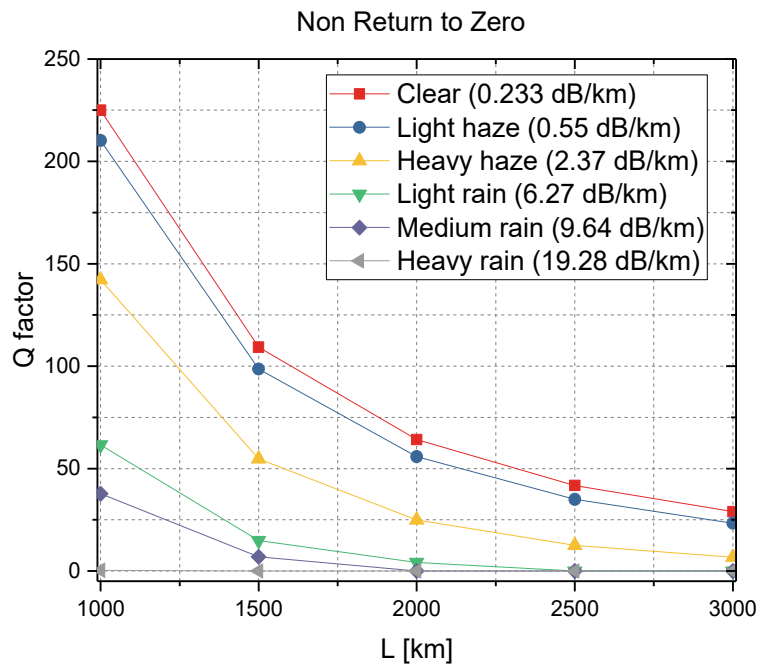

Figure $10 \mathrm{Q}$ factor for NRZ OOK modulation
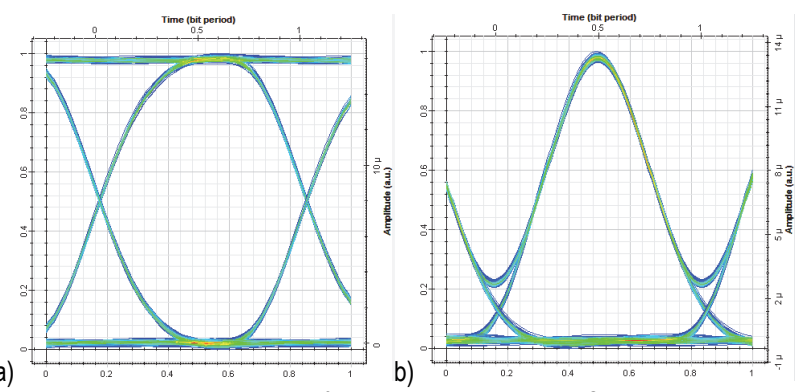

Figure $11 \mathrm{BER}$ eye diagram for attenuation caused by Clear atmospheric phenomenon for link distance $L=2000 \mathrm{~m}$ : a) RZ OOK; b) NRZ OOK
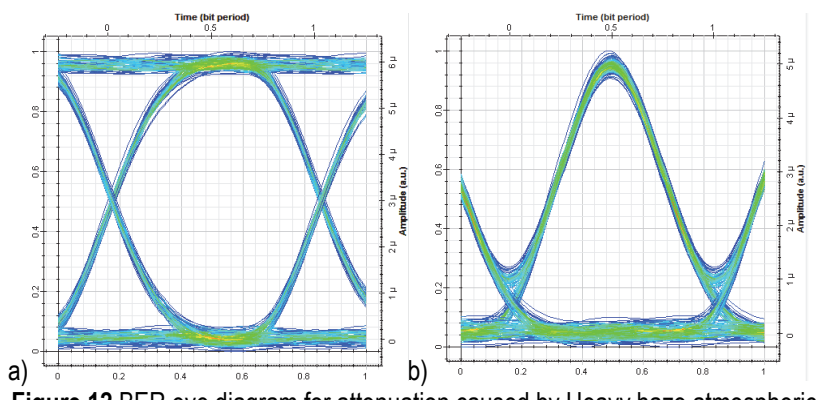

Figure 12 BER eye diagram for attenuation caused by Heavy haze atmospheric phenomenon for link distance $L=2000 \mathrm{~m}$ : a) RZ OOK; b) NRZ OOK

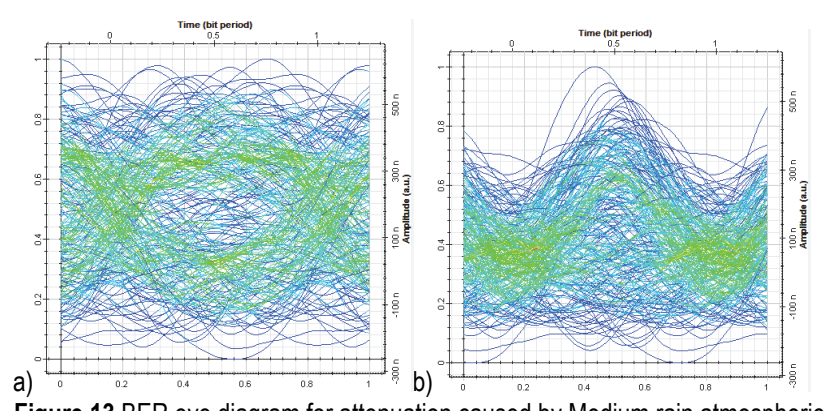

Figure 13 BER eye diagram for attenuation caused by Medium rain atmospheric phenomenon for link distance $L=2000 \mathrm{~m}$ : a) RZ OOK; b) NRZ OOK

Considering that the quality transmission is achieved if the value of $Q>5.5$. From the given figures it can be seen that the quality transmission was achieved on all link distances at Clear, Light haze and Heavy haze atmospheric phenomenon. In case of atmospheric phenomena Light rain 
and Medium rain, quality transmission can be achieved up to link distance of $2000 \mathrm{~m}$. With the atmospheric phenomena of Heavy rain, quality signal transmission is impossible to achieve for the analysed system. In that case, the source power must be increased. Also, the decrease in $Q$ factor is more pronounced at shorter distances. As the link distance increases, the decrease in the $Q$ factor is less pronounced.

From the given figures it can also be seen that the RZ OOK modulation scheme gives better results than the NRZ OOK modulation scheme. With RZ OOK modulation scheme, it can achieve quality transmission over longer distances.

\section{CONCLUSION}

In this paper, the ABER analytical expressions for digital input signals, modulated with the OOK modulation scheme, propagating in the FSO channel modelled with Gamma-Gamma, Log-Normal, $K$, and $I-K$ distributions are derived. The obtained numerical results are graphically presented in order to analyze effects of weak, moderate, and strong turbulences, as well as the length of the FSO link on the quality of signal transmission.

Based on the obtained numerical results, it can be concluded for which lengths of the FSO link and level of atmospheric turbulence which distribution model provides the best results in terms of the signal transmission with the OOK modulation scheme applied. Thus, it can be seen that for weak atmospheric turbulence, the best signal transmission results are achieved by the atmospheric channel modelled with Gamma-Gamma, $K$ and $I-K$ distributions. In the case of moderate turbulence, the best results are achieved by Log-Normal and Gamma-Gamma distributions, while in the case of weak turbulence the best results are achieved by Log-Normal, Gamma-Gamma and $I-K$ distributions. On the other hand, over shorter FSO sections, the best results are achived by Log-Normal and Gamma-Gamma distributions, while over longer sections the best results are achieved by $I-K$ and $K$ distributions (only in case of strong turbulence).

The simulation results confirm the obtained analytical and numerical results in terms of achieving transmission quality at different transmission link distances and for different atmospheric phenomena in FSO channels.

The obtained results can be used as a basis for determining other statistical characteristics to describe the transmission system operation, such as level crossing rate, phase level crossing rate, average fade duration, channel capacity, etc.

Using the presented results, it is possible to predict the behavior of the FSO system for different models of modulation schemes and in different propagation environments, which allows designers of the FSO transmission systems to provide rational systematic solutions for the desired system performance.

\section{REFERENCES}

[1] Stamatios, V. (2008). Next Generation Intelligent Optical Networks - From Access to Backbone. New York, USA: Springer.
[2] Kim, I. I., McArthur, B., \& Korevaar, E. (2001). Comparison of laser beam propagation at $785 \mathrm{~nm}$ and $1550 \mathrm{~nm}$ in fog and haze for optical wireless communications. Proc. SPIE Opt. Wireless Communications, 4214, 26-37. https://doi.org/10.1117/12.417512

[3] Wakafuji, K. \& Ohtsuki, T. (2005). Performance analysis of atmospheric optical subcarrier-multiplexing systems and atmospheric optical subcarrier-Modulated code-division multiplexing systems. Journal of Lightwave Technology, 23(4), 1676-1682. https://doi.org/10.1109/jt.2005.844500

[4] Andrews, L. C. \& Phillips, R. L. (2005). Laser beam propagation through random media,2nd ed. Bellingham, USA: SPIE Press.

[5] Anbarasi, K., Hemanth, C., \& Sangeetha, R. G. (2017). A review on channel models in free space optical communication systems. Optics \& Laser Technology, 97, 161-171. https://doi.org/10.1016/j.optlastec.2017.06.018

[6] Perić, M., Jakšić, B., Aleksić, D., Randjelović, D., \& Stefanović, M. (2018). Outage Probability of Macrodiversity Reception in the Presence Fading and Weibull Co-Channel Interference. Technical Gazette, 25(2), 376-381. https://doi.org/10.17559/TV-20161227102847

[7] Prabu, K., Kumar, D. S., \& Srinivas, T. (2014). Performance analysis of FSO links under strong atmospheric turbulence conditions using various modulation schemes. Optik, 125(19), 5573-5581. https://doi.org/10.1016/j.ijleo.2014.07.028

[8] Zhang, H., Li, H., \& Hao, C. (2016). Performance Analysis for BPSK, DPSK and OOK-Based FSO System in Atmospheric Turbulence Conditions. International Journal of Simulation - Systems, Science \& Technology, 17(36), 371376. https://doi.org/10.5013/ IJSSST.a.17.36.37

[9] Chatzidiamantis, N. D., Sandalidis, H. G., Karagiannidis, G. K., Kotsopoulos, S. A., \& Matthaiou, M. (2010). New results on turbulence modeling for free-space optical systems. 2010 17th International Conference on Telecommunications, 487492. https://doi.org/10.1109/ictel.2010.5478872

[10] Barua, B. (2011). Comparison the Performance of FreeSpace Optical Communication with OOK and BPSK Modulation under Atmospheric Turbulence. International Journal of Engineering Science. 3(5), 4391-4398.

[11] Badar, N. \& Jha, R. K. (2017). Performance comparison of various modulation schemes over free space optical (FSO) link employing Gamma-Gamma fading model. Optical and Quantum Electronics, 49(5), 192. https://doi.org/10.1007/s11082-017-1025-4

[12] Singh, J. \& Kumar, N. (2013). Performance analysis of different modulation format on free space optical communication system. Optik, 124(20), 4651-4654. https://doi.org/10.1016/j.ijleo.2013.02.014

[13] Nistazakis, H. E., Assimakopoulos, V. D., \& Tombras, G. S. (2011). Performance estimation of free space optical links over negative exponential atmospheric turbulence channels. Optik - International Journal for Light and Electron Optics, 122(24), 2191-2194. https://doi.org/10.1016/j.jileo.2011.01.013

[14] Sandalidis, H. G., Tsiftsis, T. A., \& Karagiannidis, G. K. (2009). Optical Wireless Communications With Heterodyne Detection Over Turbulence Channels With Pointing Errors. Journal of Lightwave Technology, 27(20), 4440-4445. https://doi.org/10.1109/jt.2009.2024169

[15] Dabiri, M. T., Sadough, S. M. S., \& Khalighi, M. A. (2017). FSO channel estimation for OOK modulation with APD receiver over atmospheric turbulence and pointing errors. Optics Communications, 402, 577-584. https://doi.org/10.1016/j.optcom.2017.06.077

[16] Ata, Y., Baykal, Y., \& Gökçe, M. C. (2019). Error performance of optical wireless communication systems exercising BPSK subcarrier intensity modulation in nonKolmogorov turbulent atmosphere. Optics Communications, 436, 108-112. https://doi.org/10.1016/j.optcom.2018.12.025 
[17] Balaji, K. A. \& Prabu, K. (2018). BER analysis of relay assisted PSK with OFDM ROFSO system over Malaga distribution including pointing errors under various weather conditions. Optics Communications, 426, 187-193. https://doi.org/10.1016/j.optcom.2018.05.027

[18] Singh, H. \& Sappal, A. S. (2019). Analytic and simulative comparison of turbulent FSO system with different modulation techniques. Optics \& Laser Technology, 114, 4959. https://doi.org/10.1016/j.optlastec.2019.01.013

[19] Burdah, S., Alamtaha, R., Samijayani, O. N., Rahmatia, S., \& Syahriar, A. (2019). Performance Analysis of Q Factor Optical Communication in Free Space Optics and Single Mode Fiber. Universal Journal of Electrical and Electronic Engineering, 6(3), 167-175. https://doi.org/10.13189/ujeee.2019.060311

[20]Yang, L. C. \& Huang, C. C. (2013). Performance analysis of fiber optical wide area network using SDM/WDM router. Optik, 124(14), 1700-1704. https://doi.org/10.1016/j.ijleo.2012.06.001

[21] Altınkaynak, S. \& Böcekçi, V. G. (2018). WDM Üzerinde FBG Tabanlı Sicaklık Algilama ve Performans İyileștirilmesi. (Analyzing of the Effect of Fiber Bragg Gratings on Optical Lines Using Wavelength Division Multiplexing). Marmara Fen Bilimleri Dergisi, 30(4), 460466.

[22] Gradshteyn, I. S. \& Ryzhik, I. M. (2007). Table of Integrals, Series, and Products, 7th ed. USA: Elsevier Academic Press.

[23] Yang, L., Song, X., Cheng, J., \& Holzman, J. F. (2016). FreeSpace Optical Communications Over Lognormal Fading Channels Using OOK With Finite Extinction Ratios. IEEE Access, 4, 574-584. https://doi.org/10.1109/access.2016.2520936

[24] Andrews, L. C., Phillips, R. L., \& Hopen, C. Y. (2001). Laser Beam Scintillation with Applications. Bellingham, USA: SPIE Press.

[25] Parikh, J. \& Jain, V. K. (2011). Study on statistical models of atmospheric channel for FSO communication link. 2011 Nirma University International Conference on Engineering. https://doi.org/10.1109/nuicone.2011.6153263

[26] See http://functions.wolfram.com/PDF/Erfc.pdf

[27] See http://functions.wolfram.com/PDF/MeijerG.pdf

[28] See http://functions.wolfram.com/PDF/Exp.pdf

[29] See https://optiwave.com/optisystem-overview

[30] Fadhil, H. A., Amphawan, A., Shamsuddin, H. A. B., Abd, T. H., Al-Khafaji, H. M. R., Aljunid, S.A., \& Ahmeda, N. (2013). Optimization of free space optics parameters: An optimum solution for bad weather conditions. Optik,124(19), 3969-3973. https://doi.org/10.1016/j.jileo.2012.11.059

\section{Contact information:}

\section{Jelena TODOROVIĆ, MSc}

(Corresponding author)

Faculty of Technical Sciences, University of Priština,

Knjaza Miloša 7, 38220 Kosovska Mitrovica, Serbia

E-mail: jelena.todorovic@pr.ac.rs

Branimir JAKŠIĆ, Prof. Dr.

Faculty of Technical Sciences, University of Priština, Knjaza Miloša 7, 38220 Kosovska Mitrovica, Serbia

E-mail: branimir.jaksic@pr.ac.rs

Petar SPALEVIĆ, Prof. Dr.

Faculty of Technical Sciences, University of Priština, Knjaza Miloša 7, 38220 Kosovska Mitrovica, Serbia

E-mail: petar.spalevic@pr.ac.rs

Djoko BANDJUR, Prof. Dr.

Faculty of Technical Sciences, University of Priština, Knjaza Miloša 7, 38220 Kosovska Mitrovica, Serbia

E-mail: djoko.bandjur@pr.ac.rs
Stefan PANIĆ, Prof. Dr.

Tomsk Polytechnic University,

30, Lenin Avenue, 634050 Tomsk, Russia

E-mail: stefanpnc@tpu.ru 